\title{
Pediatric Olecranon Open Reduction and Internal Fixation Utilizing Arthrex Fibertape Cerclage Suture Technique: A Case Report
}

\author{
Wesley Miaw*, James Kim, Chandler Noordhoff and Chris Miller, MD \\ Legacy Orthopedics and Sports Medicine, USA
}

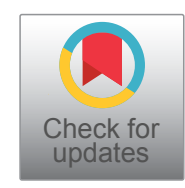

\author{
Keywords \\ Olecranon, Fracture, Arthrex fibertape, Pediatric orthopedics
}

\section{Introduction}

The elbow is a hinge-type synovial joint made up of three major bones in the arm: The humerus, radius, and ulna. The olecranon process lives on the proximal end of the ulnar bone and articulates with the olecranon fossa of the humerus, essential for extension and flexion of the joint [1]. Olecranon fractures most commonly occur with direct impact to the elbow due to minimal protection from surrounding soft tissue or with indirect impact from forceful contraction of the triceps from a fall with an outstretched arm [2]. While minor non displaced olecranon fractures may be treated non-surgically, displaced fractures most likely require surgical intervention to restore normal anatomy, extensor mechanism, and range of motion $[3,4]$. Surgical treatments may vary depending on the type of fracture presented, which typically includes the use of metal plates, screws, wires and tension bands $[3,4]$.

The Arthrex Fiber Tape Cerclage System is asolution for replacing metal cables and wires in open reduction and internal fixation (ORIF) procedures. This technique is typically used to repair displaced proximal humerus fractures. The broadened width of the suture provides better compression and load with lower complications compared to wire counterparts [57]. This technique is only indicated for simple transverse fractures, as comminuted fractures would be shortened utilizing this compression method of fixation.

\section{Case Presentation}

A 14-year-old boy presented with right elbow diffused and sharp pain following an injury playing basket ball earlier that same day. The injury occurred when the patient fell with direct trauma to his right elbow. Subsequently, the patient was admitted to a local urgent care center, where he underwent $X$-rays that included lateral, radial head, and anteroposterior views (Figure $1 \mathrm{~A}$, Figure $1 \mathrm{~B}$ and Figure $1 \mathrm{C}$ ). The patient arrived at Legacy Orthopedics and Sports Medicine for further evaluation and treatment. During the physical examination, the patient described aggravated pain with any movement and lacked the ability to actively extend his arm. Diffuse tenderness, swelling, and restricted range of motion were present. After reviewing the provided X-ray images, a diagnosis of significantly displaced, non-comminuted closed fracture of the right olecranon process was made.

\section{Surgical Technique}

The patient was placed in a prone position with adequate padding under the right shoulder and the arm abducted at about 90 degrees resting over the table (Figure 2A). A curvilinear incision was made over the posterior corner of the elbow followed by a combination of sharp, hot and blunt dissection through the skin and subcutaneous tissues to the level of the olecranon fracture. The fracture was debrided and the hemarthros is of the area was relieved and irrigated (Figure 2B). The fracture was anatomically reduced and clamped with fracture reduction clamps, which were confirmed radiographically to be anatomic. The Arthrex Fiber Tape Cerclage suture was then utilized to fixate this fracture utilizing a 2-mm drill bit and Hewson Tendon Passers (Figure 2C). Two drill tunnels were made crossing the fracture site.

The Hewson suture passer was then passed from distal to proximal through the bone, exiting proximally and then entering over the bone distally, through the bone proximally,

*Corresponding author: Wesley Miaw, Legacy Orthopedics and Sports Medicine, USA

Accepted: May 17, 2021

Published online: May 19, 2021

Citation: Miaw W, Kim J, Noordhoff C, et al. (2021) Pediatric Olecranon Open Reduction and Internal Fixation Utilizing Arthrex Fibertape Cerclage Suture Technique: A Case Report. J Orthop Surg Tech 4(1):301-305 
Citation: Miaw W, Kim J, Noordhoff C, et al. (2021) Pediatric Olecranon Open Reduction and Internal Fixation Utilizing Arthrex Fibertape Cerclage Suture Technique: A Case Report. J Orthop Surg Tech 4(1):301-305

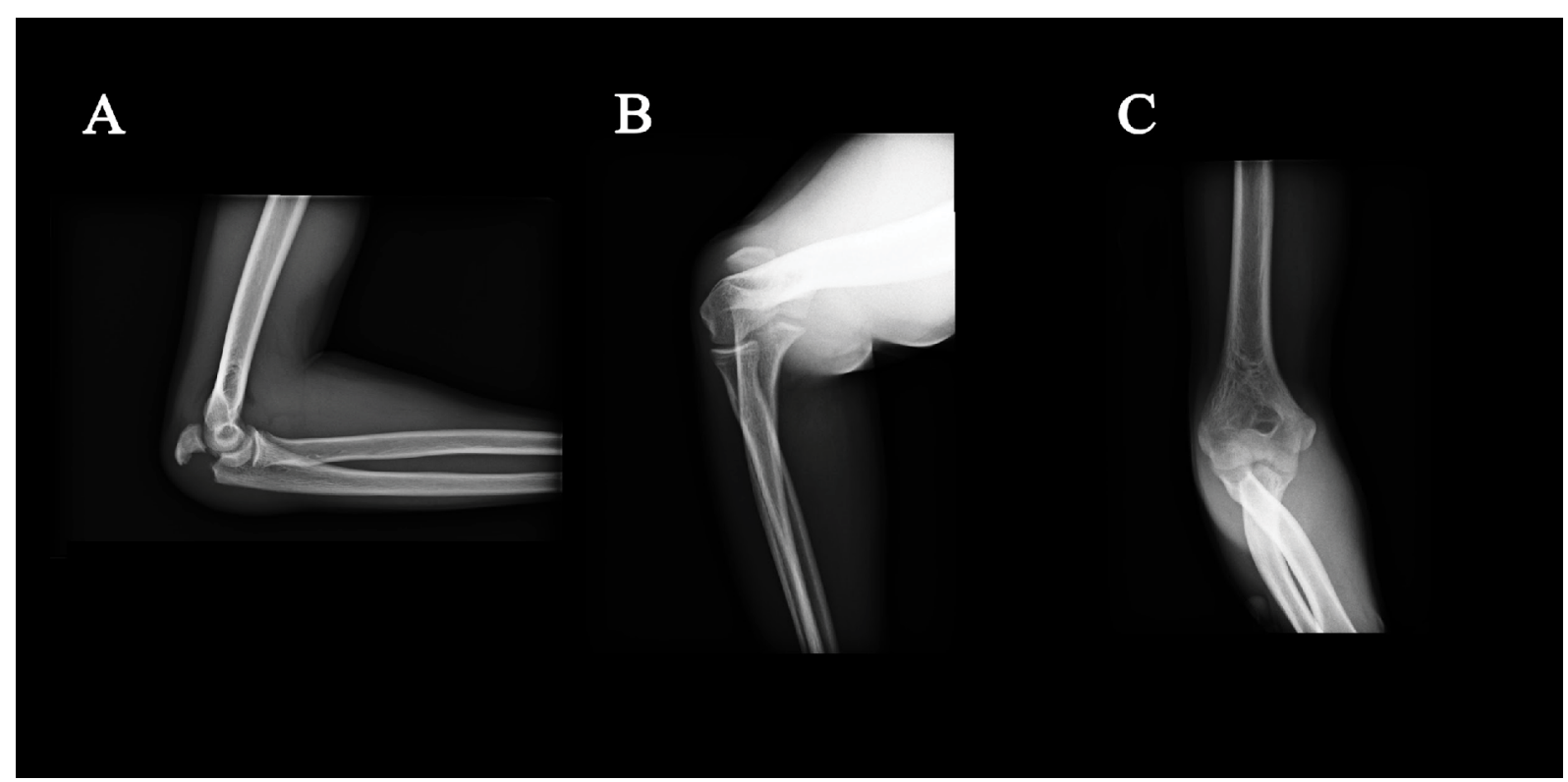

Figure 1: Initial radiographs: Right elbow closed displaced olecranon fracture (A: Lateral, B: Radial head C: Anteroposterior).

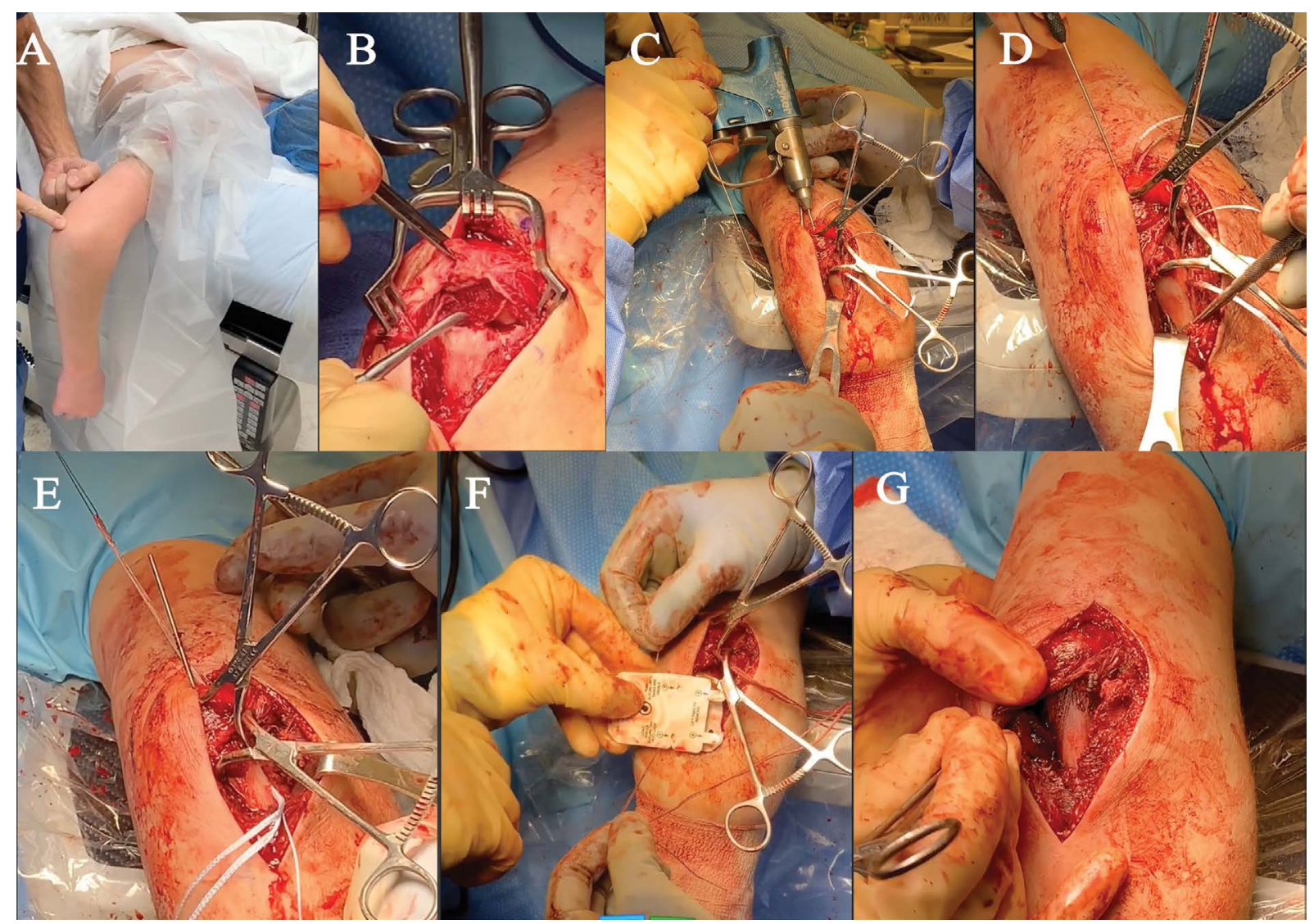

Figure 2: Open reduction and internal fixation of right olecranon process with Arthrex FiberTape Cerclage System.

and then over the bone distally, securing it into the Cerclage Arthrex system with 60-80 pounds of pressure (Figure 2D, Figure $2 \mathrm{E}$ and Figure $2 \mathrm{~F}$ ). This was confirmed visually and radiographically to be appropriate.

This was then released followed by a half-inch suture knot, and then once again tensioning and then multiple inter locking sutures to finish the fixation. A full range of motion test was performed, which confirmed the elbow to be stable with no fracture motion. Sutures were tied against the bone (Figure 2G). Additionally, the sutures were passed un- 
Citation: Miaw W, Kim J, Noordhoff C, et al. (2021) Pediatric Olecranon Open Reduction and Internal Fixation Utilizing Arthrex Fibertape Cerclage Suture Technique: A Case Report. J Orthop Surg Tech 4(1):301-305

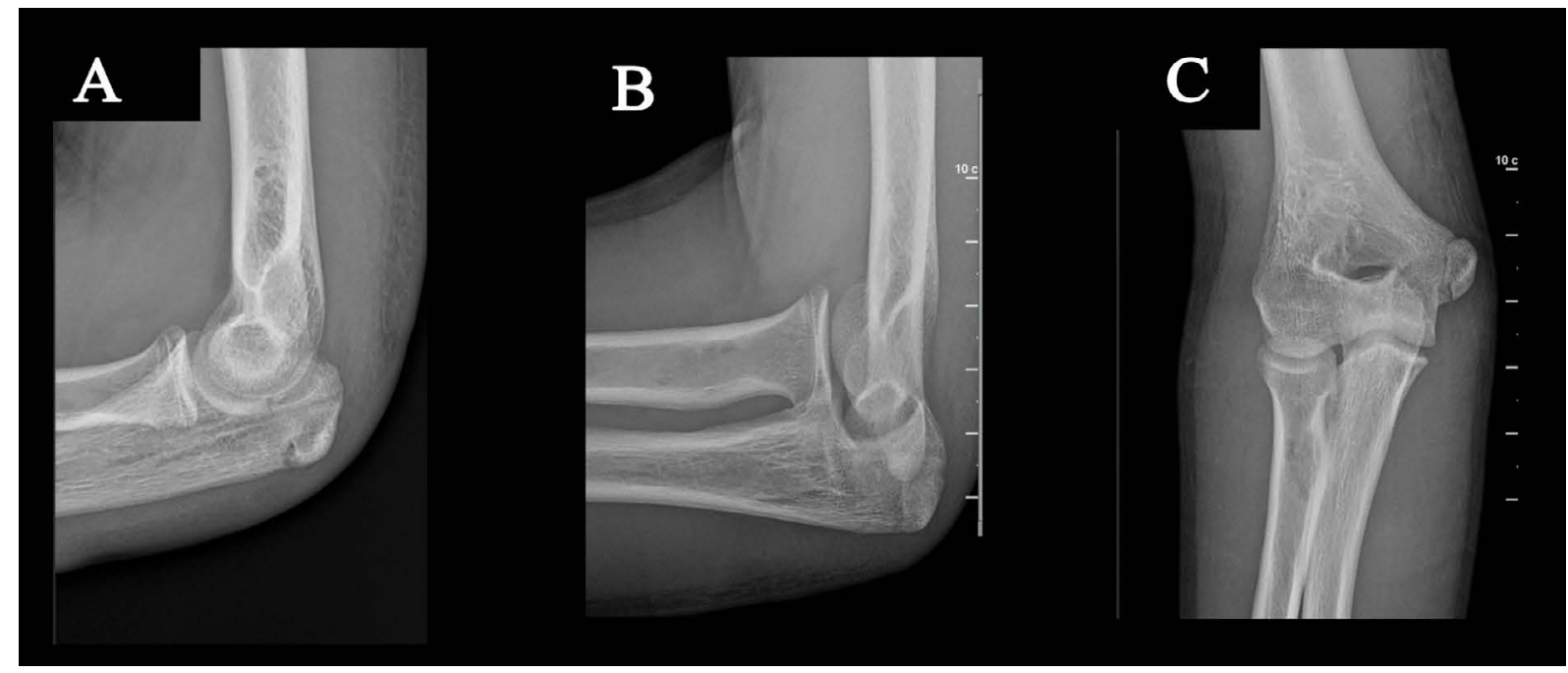

Figure 3: Radiographs directly postoperatively confirming anatomic reduction and fixation (A: Lateral, B: Radial head, C: Anteroposterior).

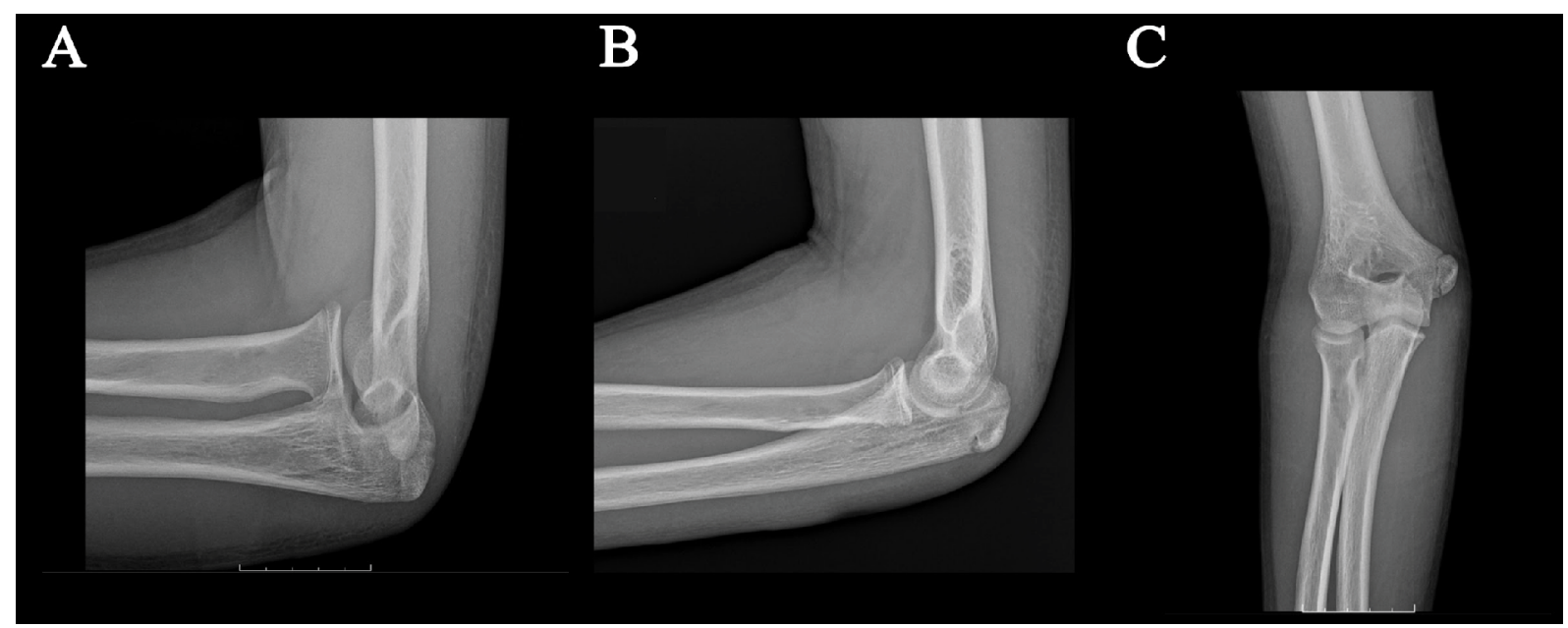

Figure 4: Radiographs at 1 week status post-procedure (A: Lateral, B: Radial head, C: Anteroposterior).

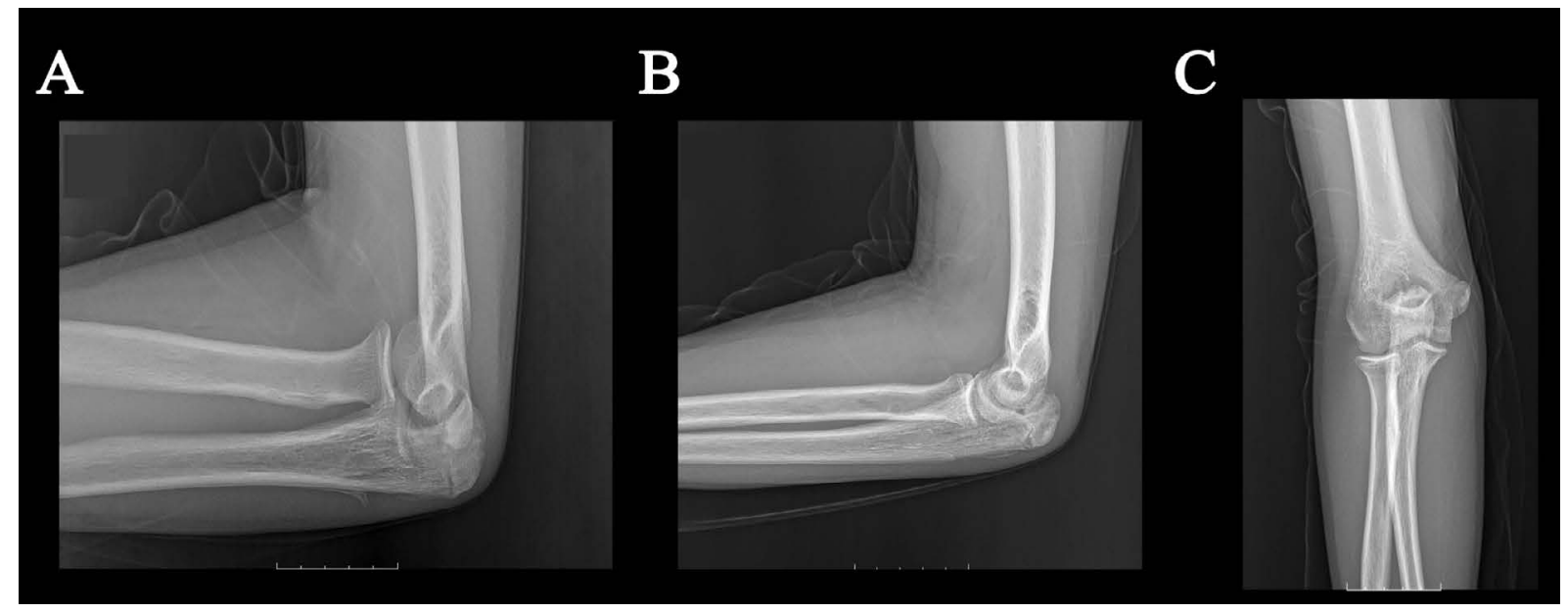

Figure 5: Radiographs at 6 weeks status post-procedure (A: Radial head, B: Lateral, C: Anteroposterior). 
Citation: Miaw W, Kim J, Noordhoff C, et al. (2021) Pediatric Olecranon Open Reduction and Internal Fixation Utilizing Arthrex Fibertape Cerclage Suture Technique: A Case Report. J Orthop Surg Tech 4(1):301-305

derneath the triceps tendon, so there were no tissue bridges. Radiographs confirmed anatomic reduction and maintenance of the fracture reduction (Figure 3A, Figure 3B and Figure 3C).

The wound was irrigated, and hemostasis was obtained. The deep fascial layer was closed with running \#2 Vicryl suture, filled by interrupted 2-0 Vicryl, 3-0 Quill and Dermabond, and a sterile compressive dressing was applied. The patient was placed in an elbow sling, postoperatively.

At the initial postoperative clinic visit 1 week status post ORIF, X-rays of the right elbow were taken with lateral, radial head, and anteroposterior views, all of which confirmed stable anatomic reduction and fixation (Figure 4A, Figure 4B and Figure 4C).

At the subsequent follow up clinic visit 6-weeks status post ORIF, X-rays of the right elbow were taken with lateral, radial head, and anteroposterior views, all of which confirmed stable anatomic reduction and fixation with healing
(Figure 5A, Figure 5B and Figure 5C).

\section{Discussion}

While olecranon fractures are relatively common in adults, they are presented less often in pediatric patients. In children, olecranon fractures only represent $4 \%$ of all elbow fractures, while the same fracture accounts for $10 \%$ of those in adults [3]. When treating these fractures, the surgeon must consider the following anatomic functions of the olecranon process. This structure is the site of insertion of the triceps muscle, which functions to extend the elbow joint. The trochlear notch of the olecranon allows articulation with the humerus. The posterior process of the olecranon prevents posterior translation of the humerus [2].

Nondisplaced olecranon fractures can be treated non-surgically with immobilization of the elbow joint at 45-90 degrees, a treatment plan that may result in diminished range of motion. When the extensor mechanism is compromised

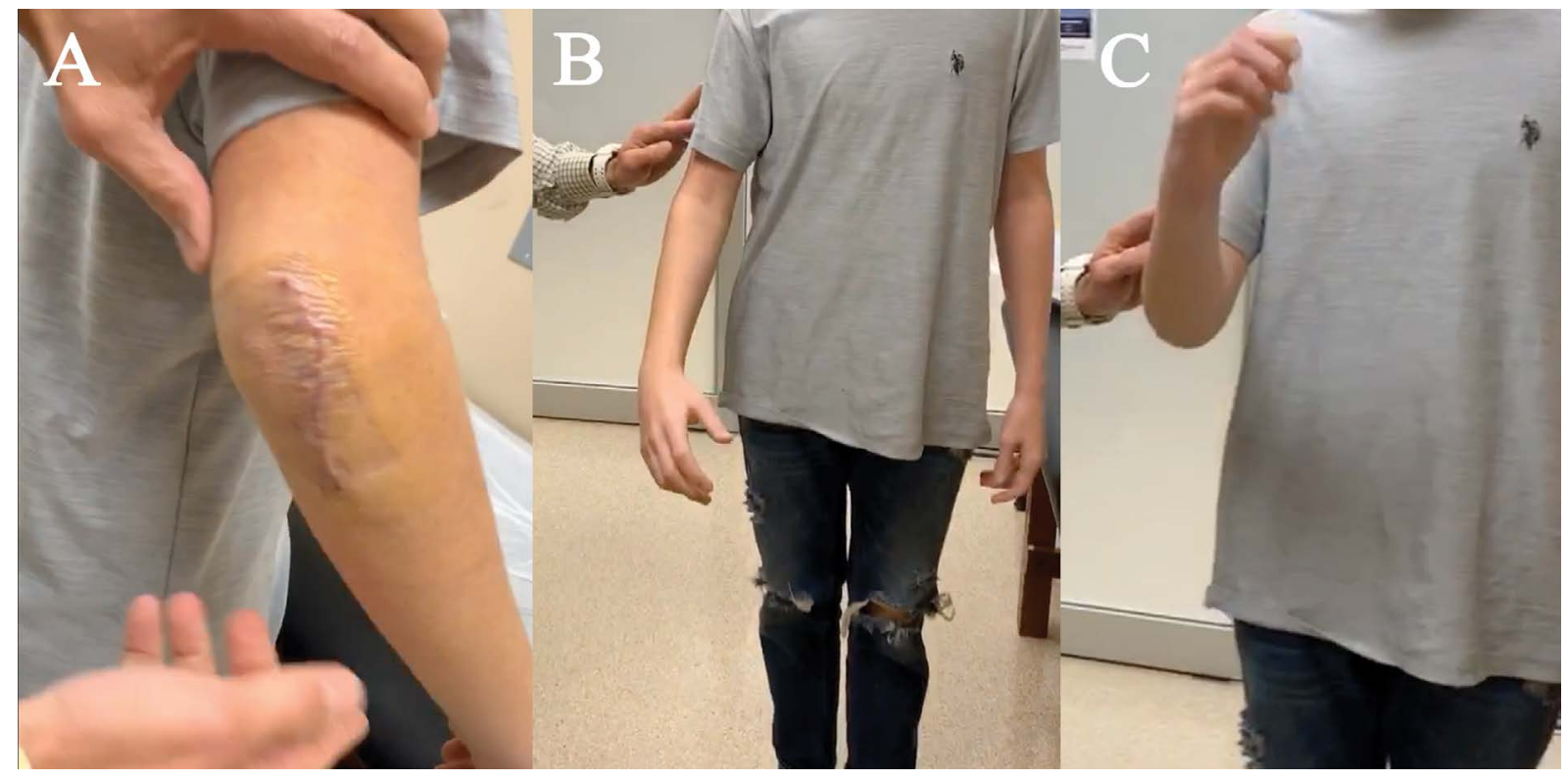

Figure 6: Physical examination at 6 days status post-procedure (A: Incision, B: Extension, C: Flexion).

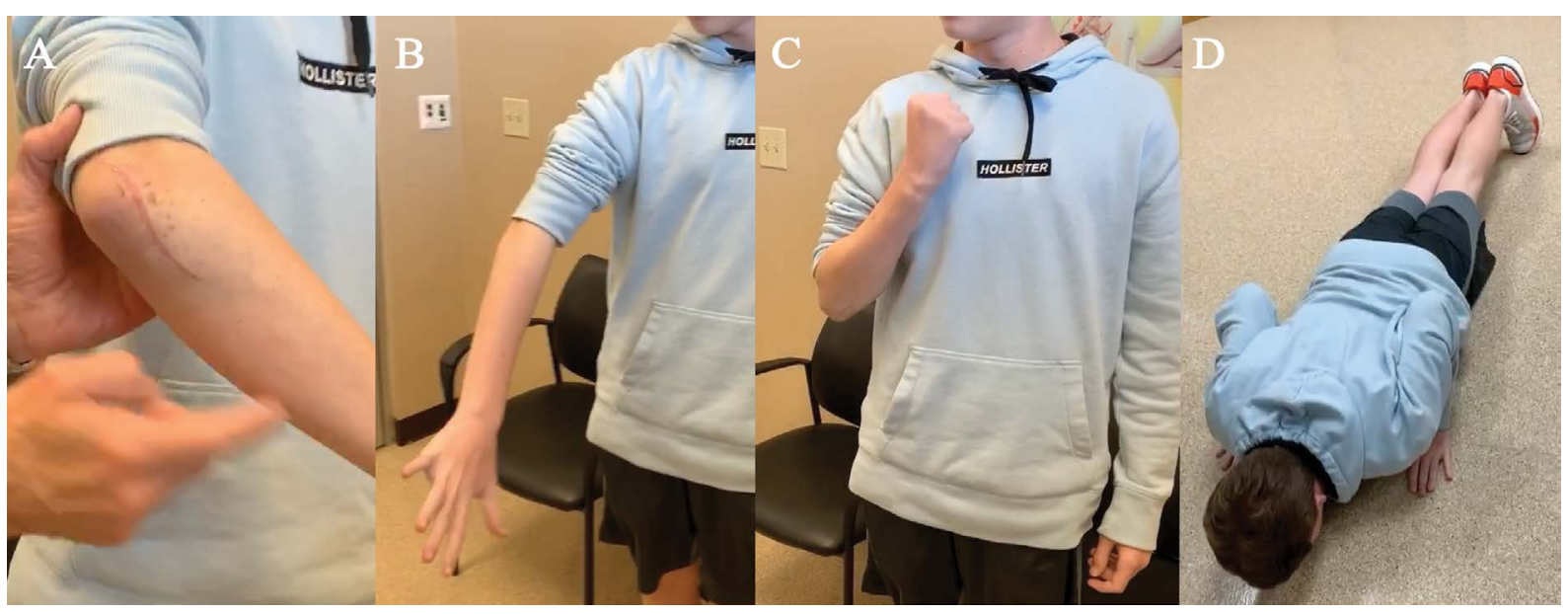

Figure 7: Physical examination 6 weeks status post-procedure (A: Incision, B: Extension and pronation, C: Flexion, D: Push up). 
Citation: Miaw W, Kim J, Noordhoff C, et al. (2021) Pediatric Olecranon Open Reduction and Internal Fixation Utilizing Arthrex Fibertape Cerclage Suture Technique: A Case Report. J Orthop Surg Tech 4(1):301-305

in displaced olecranon fractures, such as the type presented by our 14-year-old patient, surgical treatments are required for restoration of function and strength. Depending on the type of fracture, surgical interventions traditionally include tension band wiring, intramedullary screw fixation, plate fixation, or an excision and triceps advancement technique [8]. These procedures are often followed by complications involving hardware, which require a subsequent removal procedure that inherently adds risk for patients [4]. Additionally, traditional fixation techniques present a common problem of diminished range of motion post-operatively. After traditional surgical procedures, doctors typically restrict patients from stretching exercises for up to 6-weeks and place the elbow in a sling, splint, or cast, which can all result in increased stiffness [3].

The suture fixation technique utilizing the Arthrex Fiber Tape Cerclage System minimizes the need for hardware removal and improves early mobility necessary for the patient to recover full range of motion on an earlier recovery timeline. At 6-days status post ORIF surgery, our patient was cleared to discontinue his sling, begin moving his hand and elbow, and return to activities of daily livingwhile avoiding lifting of heavy objects (Figure 6). The patient's early mobility proved to help prevent stiffness in the joint and surrounding muscles. At 6 weeks status post-operation, our patient stated he felt no pain without any medication and demonstrated that he could do full body pushups, a compound movement that confirms sufficient stability and strength of the elbow joint (Figure 7). In conclusion, the innovative use of the Arthrex Fiber Tape
Cerclage System in replacement of traditional, more invasive, techniques demonstrated a treatment plan that allowed for recovery of full range of motion and stability without the need for hardware removal and associated complications.

\section{References}

1. UI Islam S, Glover A, MacFarlane RJ, et al. (2020) The anatomy and biomechanics of the elbow. The Open Orthopaedics Journal 14: 95-99.

2. Wiegand L, Bernstein J, Ahn J (2012) Fractures in brief: Olecranon fractures. Clin Orthop Relat Res 470: 3637-3641.

3. Holme TJ, Karbowiak M, Arnander M, et al. (2020) Paediatric olecranon fractures: A systematic review. EFORT Open Rev 5: 280-288.

4. Wilkerson JA, Rosenwasser MP (2014) Surgical Techniques of Olecranon Fractures. The Journal of Hand Surgery 39: 16061614.

5. https://www.arthrex.com/shoulder/fibertapesup-sup-cerclage

6. Peeters I, Depover A, Van Tongel A, et al. (2019) A review of metallic and non-metallic cerclage in orthopaedic surgery: Is there still a place for metallic cerclage? Injury 50: 1627-1633.

7. Wähnert D, Lenz M, Schlegel U, et al. (2011) Cerclage handling for improved fracture treatment. A biomechanical study on the twisting procedure. Acta Chir Orthop Traumatol Cech 78: 208214.

8. Hak David J, Golladay Gregory J (2000) Olecranon fractures: Treatment options. Journal of the American Academy of Orthopaedic Surgeons 8: 266-275.

DOI: $10.36959 / 453 / 564$ 